Draft Version February 8, 2021

Typeset using $\mathrm{LAT}_{\mathrm{E}} \mathrm{X}$ twocolumn style in AASTeX63

\title{
Gravitational-Wave Implications for the Parity Symmetry of Gravity in the High Energy Region
}

\author{
Yi-Fan Wang D ${ }^{1,2}$ Rui NiU, ${ }^{3,4}$ TaO Zhu, ${ }^{5,6}$ AND Wen ZhaO ${ }^{3,4}$ \\ ${ }^{1}$ Max-Planck-Institut für Gravitationsphysik (Albert-Einstein-Institut), D-30167 Hannover, Germany \\ ${ }^{2}$ Leibniz Universität Hannover, D-30167 Hannover, Germany \\ ${ }^{3}$ CAS Key Laboratory for Research in Galaxies and Cosmology, Department of Astronomy, University of Science and Technology of \\ China, Hefei 230026, China \\ ${ }^{4}$ School of Astronomy and Space Sciences, University of Science and Technology of China, Hefei, 230026, China \\ ${ }^{5}$ Institute for theoretical physics and Cosmology, Zhejiang University of Technology, Hangzhou, 310032, China \\ ${ }^{6}$ United center for gravitational wave physics (UCGWP), Zhejiang University of Technology, Hangzhou, 310032, China
}

\begin{abstract}
Einstein's general relativity, as the most successful theory of gravity, is one of the cornerstones of modern physics. However, the experimental tests for gravity in the high energy region are limited. The emerging gravitational-wave astronomy has opened an avenue for probing the fundamental properties of gravity in strong and dynamical field, and in particular, high energy regime. In this work, we test the parity conservation of gravity with gravitational waves. If the parity symmetry is broken, the leftand right-handed modes of gravitational waves would follow different equations of motion, dubbed as birefringence. We perform full Bayesian inference by comparing the state-of-the-art waveform with parity violation with the compact binary coalescence data released by LIGO and Virgo collaboration. We do not find any violations of general relativity, thus constrain the lower bound of the parityviolating energy scale to be $0.09 \mathrm{GeV}$ through the velocity birefringence of gravitational waves. This provides the most stringent experimental test of gravitational parity symmetry up to date. We also find third generation gravitational-wave detectors can enhance this bound to $\mathcal{O}\left(10^{2}\right) \mathrm{GeV}$ if there is still no violation, comparable to the current Large Hadron Collider (LHC) energy scale in particle physics, which indicates gravitational-wave astronomy can usher in a new era of testing the ultraviolet behavior of gravity in the high energy region.
\end{abstract}

Keywords: Gravitational Waves — Testing General Relativity — Parity Symmetry

\section{INTRODUCTION}

Symmetry is an essential characteristic of the fundamental theories of modern physics and thus necessary to be tested experimentally. In this work, we focus on the parity symmetry, which indicates the invariance of physical laws under reversed spatial coordinates. It is well-known that parity is conserved for strong and electromagnetic interactions but is broken in the weak interaction as firstly confirmed by the beta-decay experiment in cobalt-60 (Lee \& Yang 1956; Wu et al. 1957). Gravitational parity is conserved in Einstein's general relativity (GR). Nevertheless, various parity-violating grav-

yifan.wang@aei.mpg.de

wzhao7@ustc.edu.cn ity models, including Chern-Simons gravity (Alexander \& Yunes 2009), ghost-free scalar-tensor gravity (Crisostomi et al. 2018), the symmetric teleparallel equivalence of GR theory (Conroy \& Koivisto 2019) and HořavaLifshitz gravity (Hořava 2009; Wang et al. 2013) have been proposed to account for the nature of dark energy, dark matter, or quantizing gravity. In particular, in some fundamental theories of gravity, such as string theory and loop quantum gravity, the parity violation in the high energy regime is inevitable (Alexander \& Yunes 2009). Also note that, violation of gravity parity would indicate violation of Lorentz and CPT symmetry, which are expected to be conserved for all fundamental theories (Kostelecky 2004; Kostelecky \& Russell 2011; Kostelecký \& Mewes 2018).

Various astrophysical tests have put lower limits on the parity violation energy scale. Constraints from so- 
lar system test and binary pulsar observation are given by Smith et al. (2008) and Yunes \& Spergel (2009); AliHaïmoud (2011), respectively, in the context of ChernSimons gravity. However, the observational evidence for gravity in the high energy scale is limited, which leaves gravitational wave $(\mathrm{GW})$ observation as a last resort (Miller \& Yunes 2019; Yunes \& Siemens 2013; Berti et al. 2015). In contrast to the tests of the solar system or binary pulsars, GW reflects the wave behavior of the gravitational field. The tiny deviation from GR, if it exists, could be accumulated and magnified during propagation of GWs. For testing parity of gravity, Kostelecký \& Mewes (2016) inspected the peak split of GW waveform due to birefringence in general Lorentz and parity-violating gravity, and put constraints with the GW event GW150914 (Abbott et al. 2016). Nishizawa \& Kobayashi (2018) derived the GW speed for generic parity-violating gravity and constrained the violation by the GW speed measurement from GW180817/GRB170817A (Abbott et al. 2017a). A quantitative summary is given in Fig. 1 in section 4.

Our work advances in the following aspects. We first derive the $\mathrm{GW}$ waveform for generic parity-violating gravity from compact binary coalescence (CBC) using our recent results (Zhao et al. 2020b). Then the waveform is match-filtered with realistic GW data within Bayesian inference framework. This match-filtering process represents the maximum information we can extract from an individual GW event to constrain parity violation. In addition, the Bayesian approach allows us to combine constraints from multiple GW events for a tighter result. We obtain the most stringent constraints up to date for gravitational parity conservation at no higher than $0.09 \mathrm{GeV}$, which demonstrates the feasibility of probing the high energy behavior of gravity through GWs.

In what follows, we present our methods and results for inferring the constraints on parity violation in gravity from GW measurements. We first introduce the construction of the parity-violating GW waveform in the effective field theory (EFT) framework, then discuss the Bayesian inference for obtaining the constraints and the results. We also forecast the constraining ability of future ground-based GW detectors. It shows the third generation detectors such as Einstein Telescope and Cosmic Explorer can probe the parity violation energy scale to be $\mathcal{O}\left(10^{2}\right) \mathrm{GeV}$, comparable to the Large Hadron Collider (LHC) energy scale in particle physics.

\section{WAVEFORM OF GRAVITATIONAL WAVES WITH PARITY VIOLATION}

We first construct the generalized GW waveform generated by CBC with parity violation within the EFT formalism. EFT provides a systematic framework to encode all kinds of modifications to an existing theory that could arise given certain new physics, thus simultaneously testing a range of modified gravity theories at once. To investigate the possible propagation effect due to parity violation, we consider the perturbation theory of gravitational field. EFT suggests that the leadingorder modification to the linearized action of GR comes from two terms with three derivatives (Creminelli et al. 2014), i.e., $\epsilon^{i j k} \dot{h}_{i l} \partial_{j} \dot{h}_{k l}$ and $\epsilon^{i j k} \partial^{2} h_{i l} \partial_{j} h_{k l}$ with $\epsilon^{i j k}$ the antisymmetric symbol and $h_{i j}$ the tensor perturbation of metric, $\partial_{j}$ and a $\operatorname{dot}$ denote the derivatives with respect to spatial coordinates and time, respectively, $\partial^{2}$ is the Laplacian, $i, j_{\ldots}=1,2$ or 3 refer to spatial coordinate. Both terms are parity-violating. Dimensional analysis dictates that these new terms are each suppressed by an energy scale. We expect the two energy scales are of the same order, and denote collectively by $M_{\mathrm{PV}}$, which is the prime quantity we aim to constrain. Otherwise, if the two energy scales differ by orders of magnitude, only the term with lower energy scale dominates, thus we can neglect another term and our result for $M_{\mathrm{PV}}$ will not change.

Thus, in the Friedmann-Robertson-Walker (FRW) universe, choosing the unitary gauge, the linearized quadratic action of GR with leading-order parity violation is (Creminelli et al. 2014)

$$
\begin{array}{r}
S=\frac{1}{16 \pi G} \int d t d^{3} x a^{3}\left[\frac{1}{4} \dot{h}_{i j}^{2}-\frac{1}{4 a^{2}}\left(\partial_{k} h_{i j}\right)^{2}+\right. \\
\left.\frac{1}{4}\left(\frac{c_{1}}{a M_{\mathrm{PV}}} \epsilon^{i j k} \dot{h}_{i l} \partial_{j} \dot{h}_{k l}+\frac{c_{2}}{a^{3} M_{\mathrm{PV}}} \epsilon^{i j k} \partial^{2} h_{i l} \partial_{j} h_{k l}\right)\right],
\end{array}
$$

where the last two terms with three derivatives correspond to the contribution from parity violation. $c_{1}$ and $c_{2}$ are dimensionless coefficients, which are functions of cosmic time in general. As demonstrated by Alexander \& Yunes (2018), the modifications to GRbased GW waveform only arise from the propagation effect given the leading-order parity violation modification discussed above, because the generation effect occurs on a radiation-reaction timescale much smaller than the GW time of flight and its impact on the evolution of the GW waveform is negligible. We do not consider any modifications to GR quadratic terms in Eq. (1), such as Horndeski-like terms (Horndeski 1974; Nishizawa \& Kobayashi 2018; Zhao et al. 2020b) which do not violate parity symmetry. 
This EFT with leading-order extensions to GR can be mapped to all the existing specific parity-violating modified gravity models in the market. A detailed mapping dictionary can be found in Zhao et al. (2020b). Note that, in EFT, the leading-order modifications from parity-conserving terms in action are suppressed by $M_{\mathrm{PV}}^{-2}$, which indicates a much looser constraint of $M_{\mathrm{PV}}$ from GW observation (Creminelli et al. 2014; Kostelecký \& Mewes 2016; Zhao et al. 2020b). In other words, since parity violation emerges at the leading-order modification, we expect the most stringent test on gravity from the propagation of GW is on the gravitational parity symmetry from the viewpoint of EFT.

Given the parity-violating terms, the equation of motion for the GW circular polarization mode $h_{A}$ in an FRW universe is

$$
h_{A}^{\prime \prime}+\left(2+\nu_{A}\right) \mathcal{H} h_{A}^{\prime}+\left(1+\mu_{A}\right) k^{2} h_{A}=0,
$$

where $A=L$ or $R$ stands for the left- and righthand modes, $\mathcal{H}$ is the conformal Hubble parameter, $k$ is the wavenumber, a prime denotes the derivative with respect to the conformal cosmic time. Note that $\mu_{A}=\nu_{A}=0$ would reduce Eq. (2) to GR. Dimensional analysis indicates that both terms relate to the energy scale $M_{\mathrm{PV}}$ by $\mu_{A} \propto \rho_{A} k / M_{\mathrm{PV}}$ and $\nu_{A} \propto \rho_{A} k / M_{\mathrm{PV}}$ with $\rho_{R}=1$ and $\rho_{L}=-1$. The broken parity leads to asymmetry between the left- and right-hand circular polarization modes of GW during propagation. In particular, the opposite sign of $\mu_{A}$ (as well as $\nu_{A}$ ) for different modes leads to the birefringence effect of GWs, which is a characterized phenomenon for GW propagation in the parity-violating gravity. We find that the propagation of GWs can be affected in two ways. The term $\mu_{A}$ modifies the conventional dispersion relation of GWs. As a result, the velocities of left- and right-hand circular polarization of GWs are different, dubbed as the velocity birefringence of GW (Kostelecký \& Mewes 2016). On the other hand, the term $\nu_{A}$ induces the different damping rates for two polarization modes when they propagate in the expanding universe, which is called the amplitude birefringence of GWs (Alexander \& Yunes 2018). In the general parity-violating gravity theories, both effects exist. For each circular polarization mode, the former effect exactly induces the phase modifications of the GW waveform, and the latter induces the amplitude modifications. Constraints on modification of equation of motion of the same type of Eq. (2) are also obtained by LIGO and Virgo collaboration in Abbott et al. (2019a), but only parity-conserving terms are considered. In contrast, our work focus on the parity-violating effect.
The exact forms for $\mu_{A}$ and $\nu_{A}$ are (Zhao et al. 2020b)

$$
\begin{aligned}
\nu_{A} & =\left[\rho_{A} \alpha_{\nu}(\tau)\left(k / a M_{\mathrm{PV}}\right)\right]^{\prime} / \mathcal{H}, \\
\mu_{A} & =\rho_{A} \alpha_{\mu}(\tau)\left(k / a M_{\mathrm{PV}}\right),
\end{aligned}
$$

where $\tau$ is the cosmic conformal time. The functions $\alpha_{\nu} \equiv-c_{1}$ and $\alpha_{\mu} \equiv c_{1}-c_{2}$ are two arbitrary functions of time which can only be determined given a specific model of modified gravity. For GW events at the local universe, these two functions can be approximately treated as constant, i.e. ignoring their time-dependence. We also let $\alpha_{\mu}$ and $\alpha_{\nu}$ to be $\sim \mathcal{O}(1)$ by absorbing the order of magnitude into $M_{\mathrm{PV}}$.

The explicit parity-violating GW waveform can be derived from solving the equation of motion. Converting the left- and right-hand GW polarization modes into the plus and cross modes which are used more often in GW data analysis, the parity-violating waveform is

$$
\begin{aligned}
& h_{+}^{\mathrm{PV}}(f)=h_{+}^{\mathrm{GR}}(f)-h_{\times}^{\mathrm{GR}}(f)(i \delta h-\delta \Psi), \\
& h_{\times}^{\mathrm{PV}}(f)=h_{\times}^{\mathrm{GR}}(f)+h_{+}^{\mathrm{GR}}(f)(i \delta h-\delta \Psi) .
\end{aligned}
$$

The amplitude and phase modifications to the GR-based waveform $h^{\mathrm{GR}}(f)$ due to birefringence take the following parametrized form

$$
\delta h(f)=-A_{\nu} \pi f, \quad \delta \Psi(f)=A_{\mu}(\pi f)^{2} / H_{0},
$$

where $H_{0}$ is the Hubble constant. The coefficients $A_{\nu}$ and $A_{\mu}$ are given by

$$
\begin{aligned}
& A_{\nu}=M_{\mathrm{PV}}^{-1}\left(\alpha_{\nu}(z=0)-\alpha_{\nu}(z)(1+z)\right), \\
& A_{\mu}=M_{\mathrm{PV}}^{-1} \int_{0}^{z} \frac{\alpha_{\mu}\left(z^{\prime}\right)\left(1+z^{\prime}\right) d z^{\prime}}{\sqrt{\Omega_{M}\left(1+z^{\prime}\right)^{3}+\Omega_{\Lambda}}},
\end{aligned}
$$

where $z$ is the cosmic redshift. We adopt a Planck cosmology $\left(\Omega_{M}=0.315, \quad \Omega_{\Lambda}=0.685, H_{0}=\right.$ $67.4 \mathrm{~km} \mathrm{~s}^{-1} \mathrm{Mpc}^{-1}$ ) (Aghanim et al. 2020).

The above Eq. (4) represents the waveform we employ to compare with the GW data. Assume the GW is emitted at the redshift $z \sim O(0.1)$, and $\alpha_{\nu}$ and $\alpha_{\mu}$ are expected to be the same order constant, we find the ratio $\delta \Psi / \delta h \sim \pi f / H_{0} \gtrsim 10^{20}$, where $f \sim 100$ $\mathrm{Hz}$ for the ground-based GW detectors. Therefore, in the general parity-violating gravity, the corrections of GW waveform $h^{\mathrm{PV}}(f)$ mainly come from the contribution of velocity birefringence rather than that of amplitude birefringence. Note that, though we choose $\alpha_{\nu}=c_{1}-c_{2}=1$ by absorbing its order of magnitude into $M_{\mathrm{PV}}$, it is possible that $\alpha_{\nu}=0$ as is the case for a particular parity-violating gravity, the ChernSimons gravity. Nevertheless, $\alpha_{\nu}$ is non-zero for more general parity-violating gravity constructed from EFT 
(Crisostomi et al. 2018; Zhao et al. 2020b) and corresponding to ghost-free scalar-tensor gravity (Crisostomi et al. 2018), the symmetric teleparallel equivalence of GR theory (Conroy \& Koivisto 2019) or Hořava-Lifshitz gravity (Hořava 2009; Wang et al. 2013). Our main results correspond to non-zero $\alpha_{\nu}$, but we also consider the constraint for Chern-Simons gravity as a separate case, which is equivalent to let $\delta \Psi=0$ in Eq. (4).

\section{BAYESIAN INFERENCE FOR GRAVITATIONAL-WAVE EVENTS}

With the waveform Eq. (4), we can perform a direct comparison with the GW data using Bayesian inference to test the parity violation. At the time of writing, LIGO and Virgo collaborations have released the data of confident CBC events from the catalog GWTC1 (Abbott et al. 2019b) which include ten binary black hole $(\mathrm{BBH})$ events and a binary neutron star (BNS) event GW170817, together with a second BNS event, GW190425 (Abbott et al. 2020). We analyze the open data (Vallisneri et al. 2015) of the twelve events with the inference module of the open-source software PyCBC (Biwer et al. 2019) developed for GW astronomy, which in turn has dependency on LALSuite (LIGO Scientific Collaboration 2018).

Bayesian inference framework is broadly employed in GW astronomy for estimating the source parameters and selecting the preferred model from observation. Given the data $d$ of GW signal and a waveform model $H$, Bayes theorem claims

$$
P(\vec{\theta} \mid d, H, I)=\frac{P(d \mid \vec{\theta}, H, I) P(\vec{\theta} \mid H, I)}{P(d \mid H, I)},
$$

where $\vec{\theta}$ are the parameters characterizing $H, I$ is any other background information. $P(\vec{\theta} \mid H, I)$ is the prior distribution for $\vec{\theta}$ and $P(d \mid \vec{\theta}, H, I)$ is the likelihood for obtaining the data given a specific set of model parameters. The posterior $P(\vec{\theta} \mid d, H, I)$ contains all the information about the results of parameter estimation. To combine the posterior from each event to give an overall inference is also straightforward

$$
p\left(\vec{\theta} \mid\left\{d_{i}\right\}, H, I\right) \propto \prod_{i=1}^{N} p\left(\vec{\theta} \mid d_{i}, H, I\right),
$$

where $d_{i}$ denotes the $i$-th GW event.

For Gaussian and stationary noise from GW detectors, the likelihood function reads

$$
P(d \mid \vec{\theta}, H, I) \propto \exp \left[-\frac{1}{2} \sum_{i}\langle d-h(\vec{\theta}) \mid d-h(\vec{\theta})\rangle\right]
$$

where $h(\vec{\theta})$ is the GW waveform template in model $H$, and $i$ represents the $i$-th GW detector. The inner prod- uct $\langle a \mid b\rangle$ is defined to be

$$
\langle a \mid b\rangle=4 \Re \int \frac{a(f) b^{*}(f)}{S_{h}(f)} d f,
$$

where $S_{h}(f)$ is the one-side noise power spectral density (PSD) of the GW detector.

To select the model favored by observation, normalizing both sides of Eq. (7) yields the Bayes evidence

$$
P(d \mid H, I)=\int d \vec{\theta} P(d \mid \vec{\theta}, H, I) P(\vec{\theta} \mid H, I) .
$$

Bayesian ratio is defined as the ratio of evidence between two competitive models $H_{1}$ and $H_{2}$ which are GR and parity-violating gravity within this work,

$$
\mathcal{B}_{2}^{1}=\frac{P\left(d \mid H_{1}, I\right)}{P\left(d \mid H_{2}, I\right)} .
$$

The odds ratio between model $H_{1}$ and model $H_{2}$ can be expressed by

$\mathcal{O}_{2}^{1}=\frac{P\left(H_{1} \mid d, I\right)}{P\left(H_{2} \mid d, I\right)}=\frac{P\left(d \mid H_{1}, I\right)}{P\left(d \mid H_{2}, I\right)} \frac{P\left(H_{1} \mid I\right)}{P\left(H_{2} \mid I\right)}=\mathcal{B}_{2}^{1} \frac{P\left(H_{1} \mid I\right)}{P\left(H_{2} \mid I\right)}$.

Odds ratio is equal to Bayesian ratio if the competitive models are assumed to be equally likely before any measurement, which quantitatively reflects the preference of data for competitive models.

We employ the open-source software PyCBC with the open data from Vallisneri et al. (2015) to perform the Bayesian inference. For the GR waveform $h^{\mathrm{GR}}(f)$, we use the spin precessing waveform IMRPhenomPv2 (Schmidt et al. 2015; Hannam et al. 2014) when analyzing $\mathrm{BBH}$ events and spin aligned waveform with tidal deformability IMRPhenomD_NRTidal (Dietrich et al. 2017) for BNS events. The parity-violating waveform is constructed based on the above template through Eq.(4). We perform parameter estimation by selecting 16 s data for $\mathrm{BBH}$ and 200s data for BNS events to account for the relatively long signal. The data is sampled at $2048 \mathrm{~Hz}$ and the likelihood is evaluated between 20 and $1024 \mathrm{~Hz}$. The PSD is generated from 1000s data using the median estimation with 8s Hann-windowed segments and overlapped by 4s (Biwer et al. 2019). The prior is chosen to be consistent with that of Abbott et al. (2019b) and uniformly distributed for $A_{\mu}$ and $A_{\nu}$. The posterior distribution is sampled by the nest sampling algorithm dynesty (Speagle 2020) over the fiducial BBH and BNS source parameters plus the parity-violating parameters $A_{\mu}$ for velocity birefringence or $A_{\nu}$ for amplitude birefringence.

\section{RESULTS OF CONSTRAINTS ON PARITY VIOLATION}


For all the GW events, we do not find any signatures of parity violation. We thus put the lower limit of $M_{\mathrm{PV}}$ to be $0.09 \mathrm{GeV}$ in the general parity-violating gravity, which is the most stringent limit up to date. The results on $M_{\mathrm{PV}}$ are shown in Fig. 1, where we have combined the results from the twelve CBC events to give an overall constraint. For comparison, the figure also includes the results from the LAGEOS satellite in the solar system (Smith et al. 2008), that from the double pulsar system PSR J0737-3039 A/B (AliHaïmoud 2011), that from the waveform-free method using GW speed measurement from arrival time difference between GW170817 and GRB170817A (Nishizawa \& Kobayashi 2018), the expected result by considering the waveform-free method developed in Zhao et al. (2020a), and constraints from the inspection of no peak split with GW150914 in Kostelecký \& Mewes (2016).

We note that this result has direct application on constraining a range of specific parity-violating gravity models with velocity birefringence, including the ghost-free scalar-tensor gravity (Crisostomi et al. 2018), the symmetric teleparallel equivalence of GR theory (Conroy \& Koivisto 2019) and Hořava-Lifshitz gravity (Hořava 2009; Wang et al. 2013). The detailed correspondence between the above modified gravity models and the EFT formalism can be found in Ref. (Zhao et al. 2020b). The constraints can also be mapped to the standard-model extension (Kostelecky \& Russell 2011) framework for Lorentz and CPT-violated gravity, and the generalized framework for testing GR with GW propagation (Nishizawa 2018; Arai \& Nishizawa 2018; Nishizawa \& Arai 2019), but a detailed mapping relation is beyond the scope of this manuscript and thus left for future work. We have made our inference results open ${ }^{1}$ to facilitate mapping the constraint to any specific parity-violating gravity theories that one is interested in.

By similar analysis, the constraint by only considering the amplitude birefringence modification is $M_{\mathrm{PV}}>$ $1 \times 10^{-22} \mathrm{GeV}$. This result can be directly compared to and is consistent with Yagi \& Yang (2018); Alexander \& Yunes (2018); Nair et al. (2019) which focus on testing Chern-Simons gravity with GW. Compared to the constraint for general parity violation, the loose result for amplitude birefringence is because $\delta h$ is negligibly small compared to $\delta \Psi$ and the GW detection is less sensitive to amplitude modification than phase.

For analyzing the results from each individual event, we also plot Fig. 2 to show the marginalized posterior

\footnotetext{
${ }^{1}$ https://github.com/yi-fan-wang/ParitywithGW
}

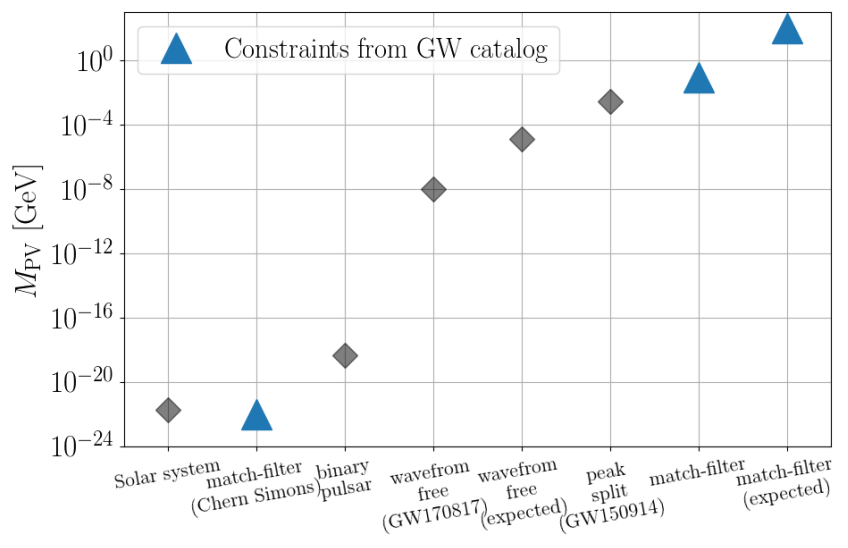

Figure 1. Constraints on the lower limits of parityviolating energy scales in gravity from various observations. The triangle markers denote the constraints by waveform match-filter with the GW catalog for the general parity-violating gravity with amplitude and velocity birefringence and the special case with only amplitude birefringence (e.g., Chern Simons gravity), respectively. The last column represents the expected constraints for general parity violation with match-filter method with the third generation ground-based GW detectors. Other existing constraints including the Solar system tests (Smith et al. 2008), the binary pulsar observation (Yunes \& Spergel 2009; Ali-Haïmoud 2011), the waveform-free result using GW speed measurements from GW170817 (Nishizawa \& Kobayashi 2018), future expected result with GW speed measurements (Zhao et al. 2020a), inspection of peak split with GW150914 (Kostelecký \& Mewes 2016) are also plotted for comparison.

distribution for $A_{\mu}$ (velocity birefringence) and Fig. 3 for the marginalized posterior distributions of $M_{\mathrm{PV}}$. From Fig. 2, we observe that the GR value $A_{\mu}=0$ is within the $90 \%$ confidence level for every event. We also report that the natural logarithm of the Bayes ratio between GR and the parity-violating gravity is in the range $[1.6,5.8]$ for all the events, confirming no parity violation for gravity.

The relatively low-mass $\mathrm{BBH}$ events, such as GW151226, GW170608, and the two BNS events give tighter constraints on $A_{\mu}$. This is because the velocity birefringence contribution corresponds to a 5.5 postNewtonian (PN) order modification to the GR waveform which has a larger impact on higher frequency, thus the low-mass events with higher cutoff frequency and longer signal yield better constraints.

In Fig. 3, for converting $A_{\mu}$ into $M_{\mathrm{PV}}$, we absorb the absolute value of $\alpha_{\mu}$ into the definition of $M_{\mathrm{PV}}$, which is equivalent to setting $\left|\alpha_{\mu}\right|=1$ in the calculation, then the parameter $M_{\mathrm{PV}}$ can be obtained from $A_{\mu}$ and redshift $z$ by Eq. (6). The combined results show that the $90 \%$ lower limit for $M_{\mathrm{PV}}$ is $0.09 \mathrm{GeV}$, representing the tightest constraint on $M_{\mathrm{PV}}$ up to date. 


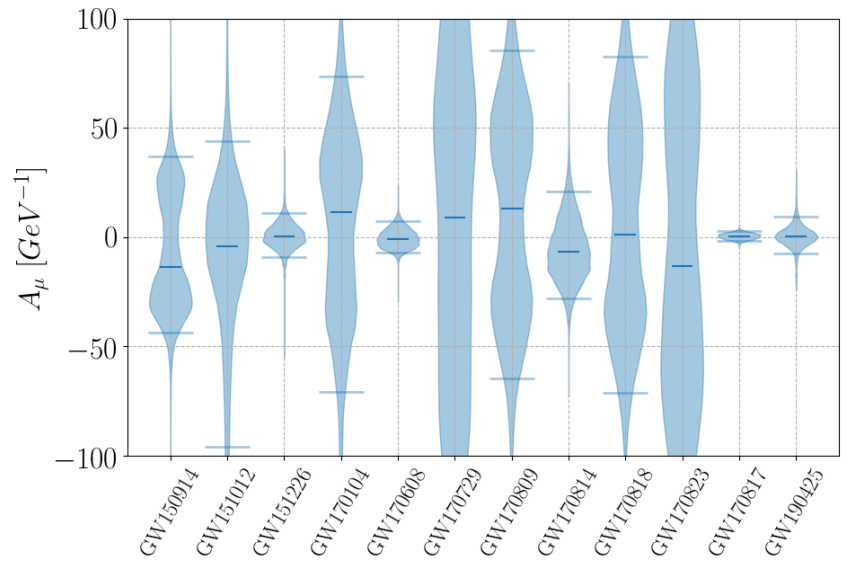

Figure 2. Violin plots of the posteriors of the parameter $A_{\mu}$ The results are obtained by analyzing the twelve GW events. The region in the posterior between the upper and lower bar denote the $90 \%$ credible interval, and the bar at the middle denotes the median value. The GR value $A_{\mu}=0$ is within the $90 \%$ confidence interval for each event. We notice that the two relative low-mass events GW151226 and GW170608 and the two BNS events yield the best constraint on $A_{\mu}$.

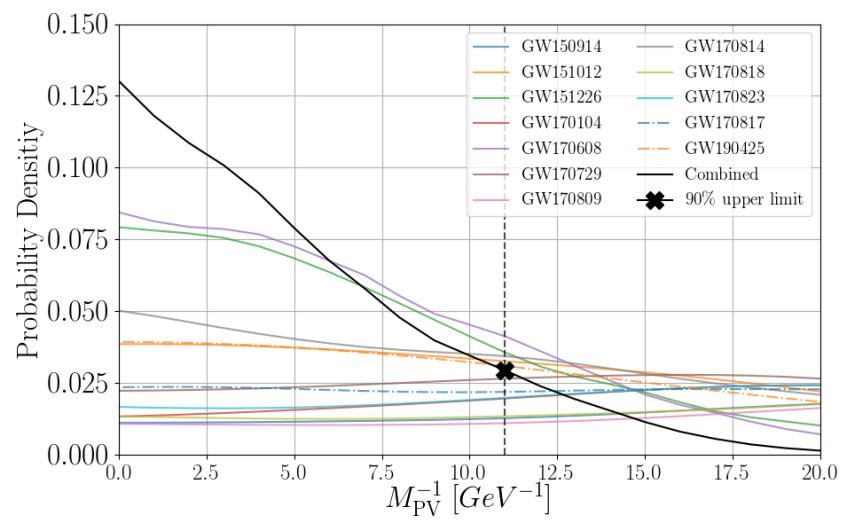

Figure 3. The posterior distributions for $M_{\mathrm{PV}}^{-1}$. The inference results for the parity-violating energy scale for velocity birefringence are plotted. The results from an individual GW event and the combination of all the events are considered. The abscissa value of the " $\mathrm{x}$ " marker represents the $90 \%$ upper limit for $M_{\mathrm{PV}}^{-1}$, or equivalently, the $90 \%$ lower limit for $M_{\mathrm{PV}}$, which is $0.09 \mathrm{GeV}$.

\section{FUTURE CONSTRAINTS WITH MORE ADVANCED DETECTORS}

With the continuing sensitivity upgrade during the advanced LIGO and Virgo runs, the future GW astronomy is even more powerful to test the parity symmetry of gravity. The KAGRA detector has joined the global network very recently. The advanced LIGO and Virgo detectors are expected to achieve the design sensitivity in a few years (Abbott et al. 2018). The third genera- tion ground-based GW detectors, including the Einstein Telescope and the Cosmic Explorer, are under projection currently (Abbott et al. 2017b). We investigate the ability of future GW astronomy to constrain the lower limit of $M_{\mathrm{PV}}$ by simulations.

We consider four sets of detector configurations based on technologies currently available or under investigation, and simulate $200 \mathrm{BBH}$ events from GR for each set. For the first set, we choose the advanced LIGO, advanced Virgo and KAGRA network, all running with designed sensitivity. The second and third sets substitute the two LIGO detectors with the 2.5 generation detector $\mathrm{A}+{ }^{2}$ and the Voyager ${ }^{3}$ configuration, respectively. The last set uses the third generation detectors including the Einstein Telescope (Punturo et al. 2010; Abbott et al. 2017b) and two Cosmic Explorer (Reitze et al. 2019; Abbott et al. 2017b) detectors located at the LIGO sites.

The simulated $\mathrm{BBH}$ events are uniformly located in the space and have mass uniformly distributed in the range $[5,50] M_{\odot}$. For the first three sets, the upper cutoff for luminosity distance is chosen to be $2000 \mathrm{Mpc}$, while for the third generation detectors the distance cutoff is $5000 \mathrm{Mpc}$. We do not consider more distant sources to give a conservative estimation of the constraining ability of the future GW detector configurations. We employ Bayesian inference to perform parameter estimation on the simulated events and choose the signal-to-noise ratio $>8$ as the criterion for detection.

In Fig. 4, we show the results of the combined constraints on $M_{\mathrm{PV}}$ with respect to the number of detections. We first notice that, out of the 200 sources, $40 \%$ of the sources can be detected by the advanced LIGO, advanced Virgo and KAGRA global network with design sensitivity, and $M_{\mathrm{PV}}$ can be constrained to $0.2 \mathrm{GeV}$, while the 2.5 generation detectors $\mathrm{A}+$ and Voyager can resolve $65 \%$ and $90 \%$ of sources, respectively, and constrain $M_{\mathrm{PV}}$ to be not less than $0.6 \mathrm{GeV}$ and $1 \mathrm{GeV}$.

For the last set of simulations, all the BBH sources can be detected by the third generation detectors, and the constraint with 200 events is $10 \mathrm{GeV}$. Given the local merger rate estimation $53.2 \mathrm{yr}^{-1} \mathrm{Gpc}^{-3}$ from LIGO and Virgo (Abbott et al. 2019c), it is expected that there are $\mathcal{O}\left(10^{4}\right) \mathrm{BBH}$ coalescence events within $5000 \mathrm{Mpc}$ in one year. Therefore, assuming the constraint on $M_{\mathrm{PV}}$ is inversely proportional to the square root of event number, the resultant constraint can reach $\mathcal{O}\left(10^{2}\right) \mathrm{GeV}$ with a one-year observation with the third generation detec-

\footnotetext{
2 https://dcc.ligo.org/LIGO-T1800042/public

${ }^{3}$ https://dcc.ligo.org/LIGO-T1500293-v11/public
} 
tors. This demonstrates the promising future of GW astronomy to probe the ultraviolet property of gravity in the high energy region, which could shed light on deviations from GR, if existing, arising from the $100 \mathrm{GeV}$ region.

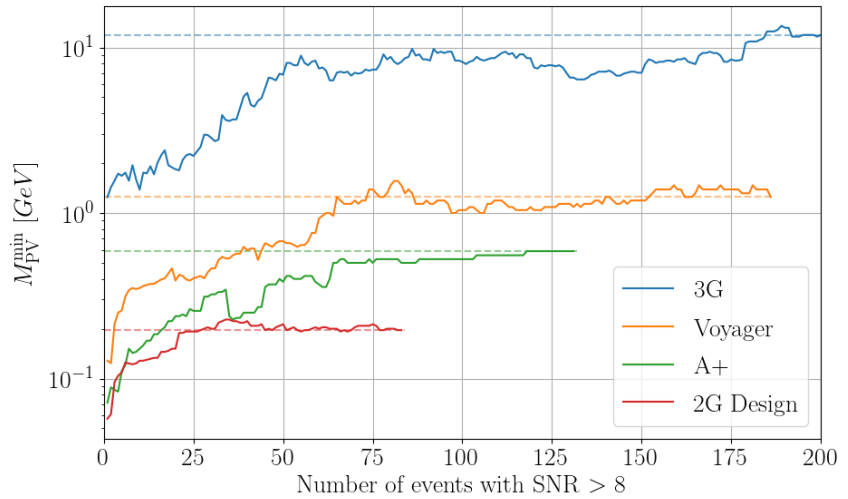

Figure 4. The projected constraints for $M_{\mathrm{PV}}$ for future GW detectors. Using $200 \mathrm{BBH}$ signals from GR, the constraints for the lower limit of the parity-violating energy scale for velocity birefringence are plotted. We consider four sets of global GW detectors network, which are: (1) the second generation detectors including advanced LIGO, advanced Virgo, and KAGRA with design sensitivity; (2) the 2.5 generation detector $\mathrm{A}+$; (3) the 2.5 generation detector Voyager; (4) the third generation detector with the Einstein Telescope and Cosmic Explorer. As the number of detections increase, the constraints for the lower limit of $M_{\mathrm{PV}}$ becomes tighter. In particular, the third generation detector can detect all the $\mathrm{BBH}$ coalescence signals within $5000 \mathrm{Mpc}$ and can constrain $M_{\mathrm{PV}}>\mathcal{O}(10) \mathrm{GeV}$ with 200 events. With a one-year observation run, the third generation detectors are expected to improve the constraint to $\mathcal{O}\left(10^{2}\right) \mathrm{GeV}$.

\section{ACKNOWLEDGMENTS}

Y.-F.W. thanks Badri Krishnan, Collin Capano and Alex Nitz for the fruitful discussion and acknowledges the Max Planck Gesellschaft for support and the Atlas cluster computing team at AEI Hannover. W.Z. and R.N. appreciate the helpful discussion with Linqing Wen, Xing Zhang, Qian Hu, Mingzheng Li and Anzhong Wang. W.Z. is supported by NSFC Grants No. 11773028, No. 11633001, No. 11653002, No. 11421303, No. 11903030, and the Strategic Priority Research Program of the Chinese Academy of Sciences Grant No. XDB23010200. T.Z. is supported in part by NSFC grants No. 11675143, the Zhejiang Provincial NSFC grants No. LR21A050001 and No. LY20A050002, and the Fundamental Research Funds for the Provincial Universities of Zhejiang in China grants No. RF- A2019015. This research has made use of data, software and/or web tools obtained from the Gravitational Wave Open Science Center (https://www.gw-openscience.org), a service of LIGO Laboratory, the LIGO Scientific Collaboration and the Virgo Collaboration. LIGO is funded by the U.S. National Science Foundation. Virgo is funded by the French Centre National de Recherche Scientifique (CNRS), the Italian Istituto Nazionale della Fisica Nucleare (INFN) and the Dutch Nikhef, with contributions by Polish and Hungarian institutes. 


\section{REFERENCES}

Abbott, B. P., et al. 2016, Phys. Rev. Lett., 116, 061102, doi: 10.1103/PhysRevLett.116.061102

Abbott, B. P., Abbott, R., Abbott, T. D., et al. 2017a, The Astrophysical Journal, 848, L13, doi: 10.3847/2041-8213/aa920c

-. 2017b, Classical and Quantum Gravity, 34, 044001, doi: 10.1088/1361-6382/aa51f4

Abbott, B. P., et al. 2018, Living Rev. Rel., 21, 3, doi: 10.1007/s41114-018-0012-9,10.1007//rr-2016-1

—. 2019a, Phys. Rev. D, 100, 104036, doi: 10.1103/PhysRevD.100.104036

—. 2019b, Phys. Rev. X, 9, 031040, doi: 10.1103/PhysRevX.9.031040

—. 2019c, Astrophys. J. Lett., 882, L24, doi: $10.3847 / 2041-8213 / a b 3800$

—. 2020, Astrophys. J. Lett., 892, L3, doi: $10.3847 / 2041-8213 / a b 75 f 5$

Aghanim, N., et al. 2020, Astron. Astrophys., 641, A6, doi: 10.1051/0004-6361/201833910

Alexander, S., \& Yunes, N. 2009, Physics Reports, 480, 1 , doi: https://doi.org/10.1016/j.physrep.2009.07.002

Alexander, S. H., \& Yunes, N. 2018, Phys. Rev. D, 97, 064033, doi: 10.1103/PhysRevD.97.064033

Ali-Haïmoud, Y. 2011, Phys. Rev. D, 83, 124050, doi: 10.1103/PhysRevD.83.124050

Arai, S., \& Nishizawa, A. 2018, Phys. Rev. D, 97, 104038, doi: 10.1103/PhysRevD.97.104038

Berti, E., Barausse, E., Cardoso, V., et al. 2015, Classical and Quantum Gravity, 32, 243001, doi: 10.1088/0264-9381/32/24/243001

Biwer, C. M., Capano, C. D., De, S., et al. 2019, Publications of the Astronomical Society of the Pacific, 131, 024503, doi: 10.1088/1538-3873/aaef0b

Conroy, A., \& Koivisto, T. 2019, Journal of Cosmology and Astroparticle Physics, 2019, 016, doi: 10.1088/1475-7516/2019/12/016

Creminelli, P., Gleyzes, J., Noreña, J., \& Vernizzi, F. 2014, Phys. Rev. Lett., 113, 231301, doi: 10.1103/PhysRevLett.113.231301

Crisostomi, M., Noui, K., Charmousis, C., \& Langlois, D. 2018, Phys. Rev. D, 97, 044034, doi: 10.1103/PhysRevD.97.044034

Dietrich, T., Bernuzzi, S., \& Tichy, W. 2017, Phys. Rev. D, 96, 121501, doi: 10.1103/PhysRevD.96.121501

Hannam, M., Schmidt, P., Bohé, A., et al. 2014, Phys. Rev. Lett., 113, 151101, doi: 10.1103/PhysRevLett.113.151101

Hořava, P. 2009, Phys. Rev. D, 79, 084008, doi: 10.1103/PhysRevD.79.084008
Horndeski, G. W. 1974, Int. J. Theor. Phys., 10, 363, doi: 10.1007/BF01807638

Kostelecky, V. 2004, Phys. Rev. D, 69, 105009, doi: 10.1103/PhysRevD.69.105009

Kostelecky, V., \& Russell, N. 2011, Rev. Mod. Phys., 83, 11, doi: 10.1103/RevModPhys.83.11

Kostelecký, V. A., \& Mewes, M. 2016, Physics Letters B, 757,510 , doi: https://doi.org/10.1016/j.physletb.2016.04.040

Kostelecký, V. A., \& Mewes, M. 2018, Phys. Lett. B, 779, 136, doi: 10.1016/j.physletb.2018.01.082

Lee, T. D., \& Yang, C. N. 1956, Phys. Rev., 104, 254, doi: 10.1103/PhysRev.104.254

LIGO Scientific Collaboration. 2018, LIGO Algorithm Library - LALSuite, free software (GPL), doi: 10.7935/GT1W-FZ16

Miller, M. C., \& Yunes, N. 2019, Nature, 568, 469, doi: 10.1038/s41586-019-1129-z

Nair, R., Perkins, S., Silva, H. O., \& Yunes, N. 2019, Phys. Rev. Lett., 123, 191101, doi: 10.1103/PhysRevLett.123.191101

Nishizawa, A. 2018, Phys. Rev. D, 97, 104037, doi: 10.1103/PhysRevD.97.104037

Nishizawa, A., \& Arai, S. 2019, Phys. Rev. D, 99, 104038, doi: 10.1103/PhysRevD.99.104038

Nishizawa, A., \& Kobayashi, T. 2018, Phys. Rev. D, 98, 124018, doi: 10.1103/PhysRevD.98.124018

Punturo, M., Abernathy, M., Acernese, F., et al. 2010, Classical and Quantum Gravity, 27, 194002, doi: 10.1088/0264-9381/27/19/194002

Reitze, D., et al. 2019, Bull. Am. Astron. Soc., 51, 035. https://arxiv.org/abs/1907.04833

Schmidt, P., Ohme, F., \& Hannam, M. 2015, Phys. Rev. D, 91, 024043, doi: 10.1103/PhysRevD.91.024043

Smith, T. L., Erickcek, A. L., Caldwell, R. R., \& Kamionkowski, M. 2008, Phys. Rev. D, 77, 024015, doi: 10.1103/PhysRevD.77.024015

Speagle, J. S. 2020, Monthly Notices of the Royal Astronomical Society, doi: 10.1093/mnras/staa278

Vallisneri, M., Kanner, J., Williams, R., Weinstein, A., \& Stephens, B. 2015, J. Phys. Conf. Ser., 610, 012021, doi: 10.1088/1742-6596/610/1/012021

Wang, A., Wu, Q., Zhao, W., \& Zhu, T. 2013, Phys. Rev. D, 87, 103512, doi: 10.1103/PhysRevD.87.103512

Wu, C. S., Ambler, E., Hayward, R. W., Hoppes, D. D., \& Hudson, R. P. 1957, Phys. Rev., 105, 1413, doi: 10.1103/PhysRev.105.1413

Yagi, K., \& Yang, H. 2018, Phys. Rev. D, 97, 104018, doi: 10.1103/PhysRevD.97.104018 
Yunes, N., \& Siemens, X. 2013, Living Reviews in Relativity, 16, doi: 10.12942/lrr-2013-9

Yunes, N., \& Spergel, D. N. 2009, Phys. Rev. D, 80, 042004, doi: 10.1103/PhysRevD.80.042004

Zhao, W., Liu, T., Wen, L., et al. 2020a, Eur. Phys. J. C, 80, 630, doi: 10.1140/epjc/s10052-020-8211-4
Zhao, W., Zhu, T., Qiao, J., \& Wang, A. 2020b, Phys. Rev. D, 101, 024002, doi: 10.1103/PhysRevD.101.024002 